\title{
Stroke in patients with SARS-CoV-2 infection: case series
}

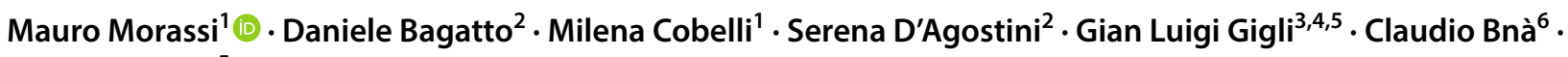 \\ Alberto Vogrig 5
}

Received: 9 April 2020 / Revised: 1 May 2020 / Accepted: 4 May 2020 / Published online: 20 May 2020

(c) Springer-Verlag GmbH Germany, part of Springer Nature 2020

\begin{abstract}
Background Italy is one of the most affected countries by the coronavirus disease 2019 (COVID-19). The responsible pathogen is named severe acute respiratory syndrome coronavirus (SARS-CoV-2). The clinical spectrum ranges from asymptomatic infection to severe pneumonia, leading to intensive care unit admission. Evidence of cerebrovascular complications associated with SARS-CoV-2 is limited. We herein report six patients who developed acute stroke during COVID-19 infection. Methods A retrospective case series of patients diagnosed with COVID-19 using reverse-transcriptase polymerase chain reaction (RT-PCR) on nasopharyngeal swabs, who developed clinical and neuroimaging evidence of acute stroke during SARS-CoV-2 infection.

Results Six patients were identified (5 men); median age was 69 years (range 57-82). Stroke subtypes were ischemic $(4,67 \%)$ and hemorrhagic $(2,33 \%)$. All patients but one had pre-existing vascular risk factors. One patient developed encephalopathy prior to stroke, characterized by focal seizures and behavioral abnormalities. COVID-19-related pneumonia was severe (i.e., requiring critical care support) in 5/6 cases (83\%). Liver enzyme alteration and lactate dehydrogenase (LDH) elevation were registered in all cases. Four patients (67\%) manifested acute kidney failure prior to stroke. Four patients (67\%) had abnormal coagulation tests. The outcome was poor in the majority of the patients: five died (83\%) and the remaining one (17\%) remained severely neurologically affected (mRS: 4).

Conclusions Both ischemic and hemorrhagic stroke can complicate the course of COVI-19 infection. In our series, stroke developed mostly in patients with severe pneumonia and multiorgan failure, liver enzymes and LDH were markedly increased in all cases, and the outcome was poor.
\end{abstract}

Keywords COVID-19 $\cdot$ Coronavirus $\cdot$ Encephalitis $\cdot$ Cerebrovascular disease $\cdot$ Neurological complications $\cdot$ Brain hemorrhage

Mauro Morassi

mauro.morassi@poliambulanza.it

1 Unit of Neuroradiology, Department of Diagnostic Imaging, Istituto Ospedaliero Fondazione Poliambulanza, Via L. Bissolati 57, 25124 Brescia, Italy

2 Unit of Neuroradiology, Department of Diagnostic Imaging, Azienda Sanitaria Universitaria Friuli Centrale, Presidio Ospedaliero Santa Maria della Misericordia, Udine, Italy

3 Department of Medicine (DAME), University of Udine Medical School, Udine, Italy

4 Department of Mathematics, Informatics and Physics (DMIF), University of Udine, Udine, Italy

5 Clinical Neurology Unit, Azienda Sanitaria Universitaria Friuli Centrale, Presidio Ospedaliero Santa Maria della Misericordia, Udine, Italy

6 Unit of Radiology, Department of Diagnostic Imaging, Istituto Ospedaliero Fondazione Poliambulanza, Brescia, Italy

\section{Introduction}

Italy was the first country in Europe to witness an outbreak of the coronavirus disease 2019 (COVID-19), which, as of April 6, has infected 1,277,196 people worldwide [1]. The responsible pathogen is named severe acute respiratory syndrome coronavirus (SARS-CoV-2). The clinical spectrum associated with COVID-19 ranges from asymptomatic infection to severe pneumonia, leading to intensive care unit (ICU) admission [2-4]. Evidence of cerebrovascular complications associated with SARS-CoV-2 is limited, but previous reports from the SARS epidemic in Asia in 2003 suggested a higher incidence of thromboembolic complications, including stroke [5]. Management of acute stroke in the setting of this pandemic disease poses unique challenges, including the need to maintain high-quality stroke care with limited 
resources, while at the same time preventing infection spread to patients and physicians [6]. A better characterization of cerebrovascular complications associated with COVID-19 infection is important to guide decision making.

We herein report the clinical spectrum, neuroimaging findings, and outcome of six patients developing acute stroke during COVID-19 infection.

\section{Methods}

This is a retrospective observational case series of patients developing clinical and neuroimaging evidence of acute stroke during COVID-19 infection. We included only patients with laboratory-confirmed COVID-19 infection who were admitted to the Fondazione Poliambulanza Hospital, Brescia, Italy, or the Udine University Hospital, Udine, Italy, between March 16 and April 5, 2020. A confirmed case of COVID-19 was defined by a positive result on a reverse-transcriptase polymerase chain reaction (RT-PCR) assay on nasopharyngeal swabs. Demographic data, information on clinical presentation and evolution, and laboratory and radiologic results were recorded. The severity of acute respiratory distress syndrome (ARDS) was rated using the $\mathrm{PaO}_{2} / \mathrm{FiO}_{2}$ ratio (partial pressure of arterial oxygen over the fraction of inspired oxygen) which was indicated whenever available. Acute lung injury is present if $\mathrm{PaO}_{2} / \mathrm{FiO}_{2}$ ratio is $\leq 300$ [7]. Each diagnosis of stroke was confirmed by brain computed tomography (CT)/CT angiography (CTA), and/or magnetic resonance imaging (MRI). All examinations were reviewed by two experienced neuroradiologists (DB, MM). Neurological presentations were reviewed by one experienced neurologist (AV). Descriptive analysis is presented as frequencies and percentages for categorical variables and as the median and range for continuous variables. All procedures were performed in accordance with the institutional ethics committee and the Declaration of Helsinki.

\section{Results}

Six patients were identified ( 5 men); median age was 69 years (range 57-82). Stroke subtypes were ischemic (4, $67 \%)$ and hemorrhagic $(2,33 \%)$. All patients but one had pre-existing vascular risk factors. COVID-19-related pneumonia was severe (i.e., requiring critical care support) in $5 / 6$ cases $(83 \%)$. Liver enzyme alteration and lactate dehydrogenase elevation were registered in all cases $(100 \%)$. Four patients (67\%) manifested acute kidney failure prior to stroke. Four patients $(67 \%)$ had abnormal coagulation tests. Cerebrovascular complications were multiple and bilateral in four cases $(67 \%)$. The outcome was poor in the majority of the patients: five died (83\%), and the remaining one (17\%) remains severely neurologically affected (mRS: 4).

Table 1 Characteristics of patients with cerebrovascular complications of SARS-CoV-2 infection

\begin{tabular}{|c|c|c|c|c|c|c|c|c|}
\hline $\begin{array}{l}\text { Case no. (age, } \\
\text { sex) }\end{array}$ & Stroke type & $\begin{array}{l}\text { Vascular risk } \\
\text { factors }\end{array}$ & $\begin{array}{l}\text { SARS- } \\
\text { CoV-2 } \\
\text { involvement }\end{array}$ & Kidney failure & $\begin{array}{l}\text { Liver } \\
\text { enzyme } \\
\text { alteration }\end{array}$ & $\begin{array}{l}\text { Increased } \\
\text { LDH }\end{array}$ & $\begin{array}{l}\text { Abnormal blood } \\
\text { clotting tests }\end{array}$ & Outcome \\
\hline $1(64, M)$ & $\begin{array}{l}\text { Ischemic (throm- } \\
\text { boembolic) }\end{array}$ & $\begin{array}{l}\text { Previous smoker, } \\
\text { history of } \\
\text { myocardial } \\
\text { infarction }\end{array}$ & Severe & + & + & + & $\begin{array}{l}+(\uparrow \text { aPTT, } \\
\uparrow \text { INR, } \uparrow \\
\text { D-dimer, } \downarrow \\
\text { platelet count })\end{array}$ & Death \\
\hline $2(75, M)$ & $\begin{array}{l}\text { Ischemic (throm- } \\
\text { boembolic) }\end{array}$ & $\begin{array}{l}\text { Arterial hyper- } \\
\text { tension, diabe- } \\
\text { tes mellitus }\end{array}$ & Severe & + & + & + & $+(\uparrow \mathrm{INR})$ & Death \\
\hline $3(82, M)$ & Ischemic & $\begin{array}{l}\text { Arterial hyper- } \\
\text { tension, dia- } \\
\text { betes mellitus, } \\
\text { and previous } \\
\text { TIA }\end{array}$ & Severe & + & + & + & No & Death \\
\hline $4(76, F)$ & Ischemic & $\begin{array}{l}\text { Arterial hyper- } \\
\text { tension, dia- } \\
\text { betes mellitus, } \\
\text { aortic valve } \\
\text { replacement, } \\
\text { previous stroke }\end{array}$ & Moderate & No & + & + & $+(\uparrow \mathrm{D}$-dimer $)$ & $\mathrm{mRS}=4$ \\
\hline $5(57, M)$ & Hemorrhagic & $\begin{array}{l}\text { Arterial hyper- } \\
\text { tension, throm- } \\
\text { bocytosis }\end{array}$ & Severe & + & + & + & $\begin{array}{c}+(\uparrow \mathrm{aPTT}, \uparrow \\
\mathrm{D} \text {-dimer })\end{array}$ & Death \\
\hline $6(57, M)$ & Hemorrhagic & None & Severe & No & + & + & No & Death \\
\hline
\end{tabular}


Clinical and laboratory data for all patients are summarized in Table 1.

\section{Case 1}

A 64-year-old man presented with cough, fever, and dyspnea for 6 days. He had a previous history of smoking, but he was not a current smoker. Due to an acute myocardial infarction, he underwent a percutaneous coronary intervention 13 years earlier. His treatment included dual antiplatelet therapy (aspirin and clopidogrel) and ramipril. Body temperature at admission was $38.6{ }^{\circ} \mathrm{C}$. Clinical and laboratory findings indicated moderate respiratory distress $\left(\mathrm{PaO}_{2} / \mathrm{FiO}_{2}\right.$ 238). Chest $X$-ray demonstrated bilateral areas of consolidation. The respiratory function progressively worsened and 3 days later he was intubated and mechanically ventilated. In the following days, the patient developed kidney failure (creatinine $3.22 \mathrm{mg} / \mathrm{dL}$, n.v. 0.72-1.18), increased indices of inflammation and hepatic injury (C-reactive protein [CRP] $175 \mathrm{mg} / \mathrm{L}$, n.v. < 5; lactate dehydrogenase [LDH] $1032 \mathrm{IU} / \mathrm{L}$, n.v. 125-220; aspartate aminotransferase [AST] $152 \mathrm{IU} / \mathrm{L}$, n.v. 4-40; gamma-glutamyltransferase [GGT] 1165 IU/L, n.v. 8-61; total bilirubin $6.64 \mathrm{mg} / \mathrm{dL}$, n.v. $0.2-1$ ) and neurological deterioration, with inability to arouse during sedation lightening. All these features together were suggestive of multiorgan failure. Coagulation tests showed thrombocytopenia $\left(78 \times 10^{3} / \mu \mathrm{L}\right.$, n.v. $\left.150-400\right)$ associated with increased international normalized ratio (INR 1.62, n.v. $0.80-1.20$ ) and increased D-dimer (7744 ng/mL, n.v. 0-500). On day 16, a total body CT demonstrated extensive bilateral consolidations and ground-glass opacities of the lungs with bilateral pleural effusion (Fig. 1a) and a filling defect in the right pulmonary artery due to pulmonary embolism. A cuneiform hypodense area was also detected in the spleen (Fig. 1b). Brain CT showed multiple hypodense lesions involving different cortical and subcortical regions of both cerebral hemispheres (Fig. 1c and d). Clinical, laboratory and imaging findings were consistent with pulmonary thrombo-embolism associated with multiple ischemic lesions involving the brain and spleen. Three days after CT examination, the patient died.

\section{Case 2}

A 75-year-old man with diabetes mellitus and arterial hypertension presented with cough and fever for 5 days. His treatment included metformin and anti-hypertensive drugs (enalapril and hydrochlorothiazide). Body temperature at admission was $38.9^{\circ} \mathrm{C}$. Clinical and laboratory findings indicated mild respiratory distress $\left(\mathrm{PaO}_{2} / \mathrm{FiO}_{2} 347\right)$. Chest $\mathrm{CT}$ demonstrated bilateral ground-glass opacities (Fig. 1e). On day 12 , the respiratory function worsened and the patient was found unconscious with severe hypoxemia $\left(\mathrm{SO}_{2} 76 \%\right)$ and left-sided hemiparesis. Brain CT and CTA showed cortico-subcortical hypodensity over the right cingulate gyrus (Fig. 1f-g) with occlusion of the right pericallosal

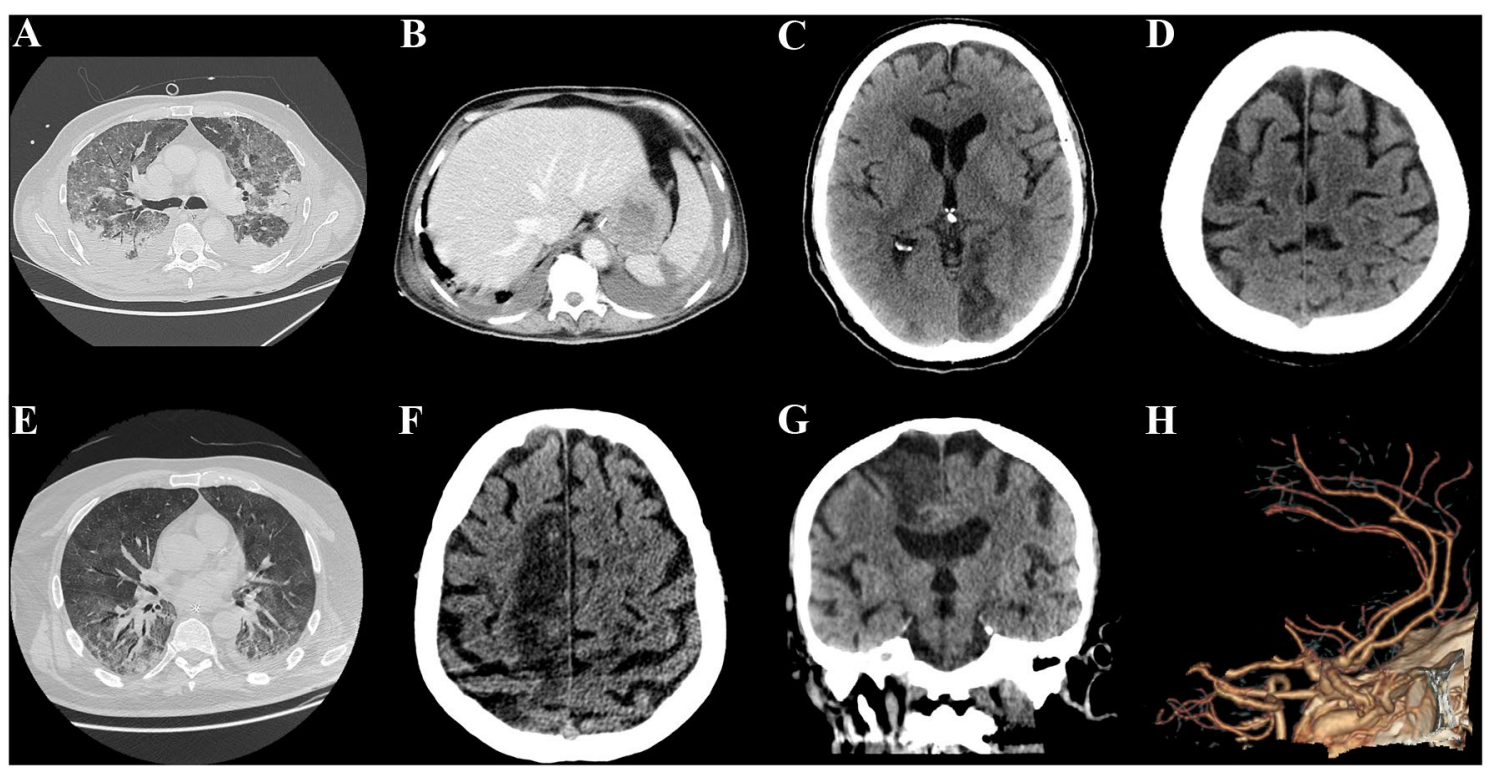

Fig. 1 Total body and brain CT examination of Patient 1 a-d showing extensive bilateral consolidations and ground-glass opacities of the lungs (a), a hypodense area in the upper portion of the spleen (b) and two different ischemic lesions involving the left occipital lobe (c) and the right frontal precentral gyrus (d), respectively. Chest and brain CT examination of Patient $2 \mathbf{e}-\mathbf{h}$ showing bilateral consolida- tions and ground-glass opacities of the lungs with pleural effusion on the left side (e), an ischemic lesion involving the frontal lobe on the right side (f-g), and the occlusion of the right pericallosal artery on a 3D-volume rendering reconstruction of the brain CT angiography examination (h) 
artery (Fig. 1h). The same day, the patient was transferred to ICU where he was intubated and mechanically ventilated. Blood tests were significant for increased CRP $(46 \mathrm{mg} / \mathrm{L}$, n.v. $<5)$, creatinine $(5 \mathrm{mg} / \mathrm{dL})$, liver enzymes (alanine aminotransferase [ALT] 74 IU/L, n.v. <60), LDH (979 IU/L, n.v. 125-220), and INR (INR 1.53, n.v. 0.80-1.20). On day 21, the patient's neurological status further declined, with a Glasgow Coma Scale (GCS) of 3. New brain CT showed multiple, bilateral, supratentorial (right frontal and parietal, left perirolandic and bilateral occipital) and infra-tentorial (vermian, left cerebellar hemisphere) ischemic lesions. Chest CT showed extension of ground-glass opacities and consolidations (involving about $75 \%$ of the lung parenchyma). Electroencephalogram (EEG) demonstrated bilateral slowing of the background rhythm with frontal sharp waves. On day 38, he developed hemodynamic instability and died.

\section{Case 3}

An 82-year-old man presented with cough, fever, and dyspnea for 7 days. He had a previous history of mild diabetes mellitus, hypertensive cardiomyopathy, aortic valve regurgitation, and previous transitory ischemic attack. His treatment included aspirin and telmisartan, but not insulin. In 2015, he underwent left carotid endarterectomy. Body temperature at admission was $37.9^{\circ} \mathrm{C}$. Clinical and laboratory evaluation showed moderate respiratory distress $\left(\mathrm{PaO}_{2} / \mathrm{FiO}_{2} 198\right)$, increased creatinine (1.22 mg/dL, n.v. 0.72-1.18), CRP
(181 mg/L, n.v. <5), and LDH (507 IU/L, n.v. 125-220). Coagulation tests were normal. Chest radiography demonstrated hazy ground-glass opacities involving the peripheral and basal regions of the lungs with no significant pleural effusion (Fig. 2a). The next day, he developed left-sided hemiparesis. Brain CT examination showed a new small hypodense area in the right thalamus of presumed ischemic origin (Fig. 2b, initial imaging; c, with ischemic lesion). CT-angiography showed no intra-luminal filling defect in the carotid arteries, vertebrobasilar system, and other intra-cranial vessels. During hospitalization, he was treated with dual antiplatelet therapy (aspirin and clopidogrel) together with enoxaparin. On day 3, a new brain CT showed appearance of an additional small hypodense area in the centrum semiovale (Fig. 2d). No intra-axial hemorrhages were detected. On day 4 , the patient rapidly became comatose and died.

\section{Case 4}

A 76-year-old woman presented with cough and fever for 10 days. The patient had a previous history of arterial hypertension, diabetes mellitus, aortic valve replacement, and cerebellar stroke. Her treatment included aspirin and warfarin. Body temperature at admission was $37.6{ }^{\circ} \mathrm{C}$. Clinical and laboratory evaluation indicated mild respiratory distress $\left(\mathrm{PaO}_{2} / \mathrm{FiO}_{2} 261\right)$. Chest CT demonstrated multiple areas of ground-glass opacities and consolidations mainly involving the lower lobes (Fig. 2e). The family members reported a

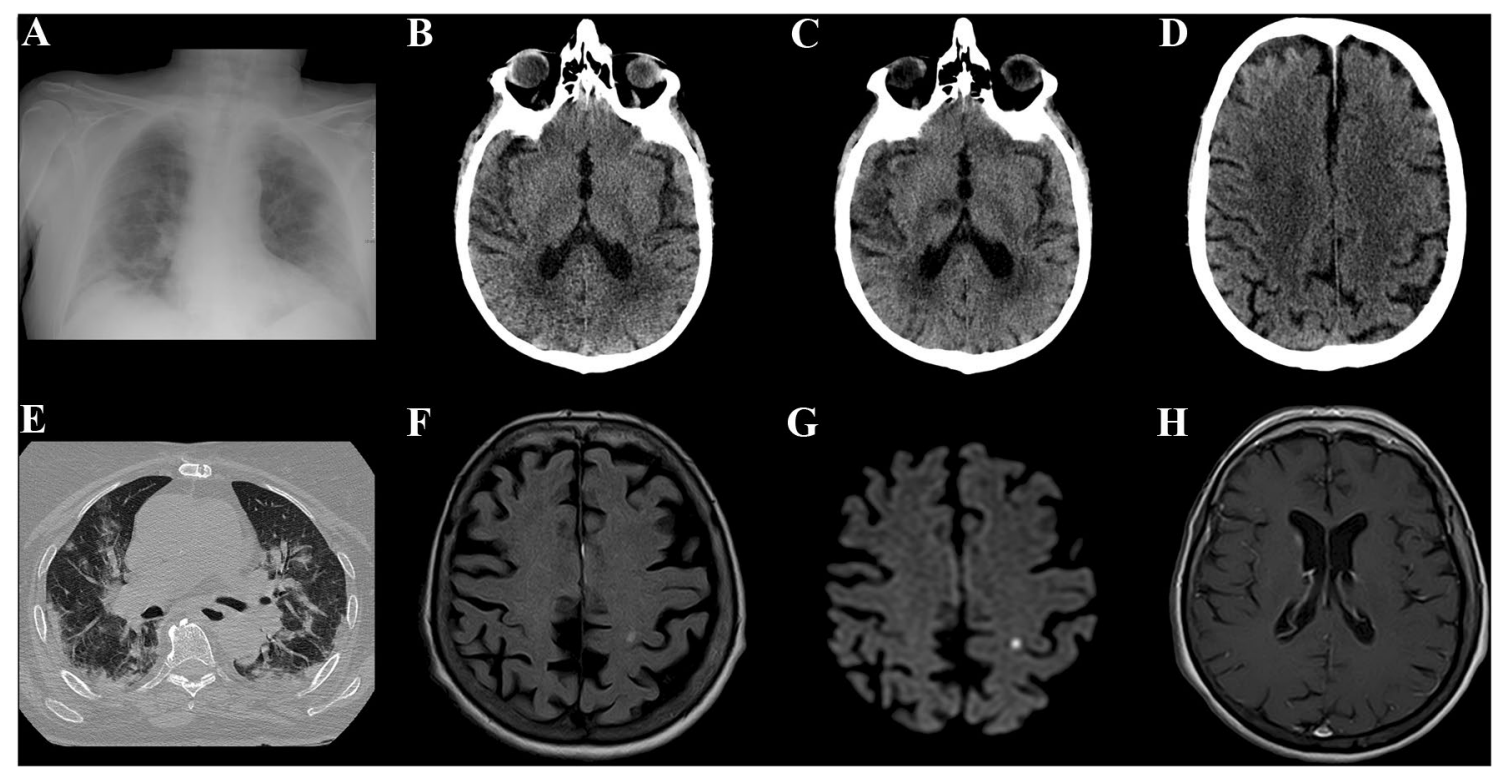

Fig. 2 Chest X-ray and two different brain CT examinations of Patient 3 a-d showing multiple opacities involving both lungs and a left lower lobe consolidation (a), the normal aspect of both thalami (b) and the appearance few days later of multiple ischemic lesions involving the right thalamus (c), and the subcortical white matter of the centrum semiovale of the same side (d). Chest CT and brain
MRI examinations of patient $4 \mathbf{e}-\mathbf{h}$, demonstrating multiple areas of ground-glass opacities and consolidations mainly involving the lower lobes (e), a focal T2-FLAIR hyperintensity lesion in the left precentral gyrus (f) with a bright signal on DWI sequence (g), and mild post-contrast enhancement of the head of the right caudate nucleus (h) 
number of episodes of transient loss of consciousness, followed by confusion, in the days preceding admission to the hospital. Blood tests showed increased CRP (12 mg/L, n.v. <5) and D-dimer (1381 ng/mL, n.v. 0-500). On day 4, EEG showed a normal background in the alpha range $(8 \mathrm{~Hz})$, associated with recurrent sharp slow waves over the left temporal region, which occasionally were seen also on the right homologous regions. The episodes were interpreted as focal seizures and treatment with levetiracetam was introduced. A brain CT demonstrated a small hypodense area $(5 \mathrm{~mm})$ in the head of the right caudate nucleus referable to a lacunar infarction. On day 5, a contrast-enhanced brain MRI showed a small rounded area of diffusion restriction on the left prerolandic gyrus (Fig. 2f, fluid attenuated inversion recovery [FLAIR] imaging; g, diffusion-weighted imaging [DWI]). The lesion previously noted in the right caudate nucleus was hyperintense on T2-FLAIR, with no diffusion restriction but faint contrast enhancement (Fig. 2h). A small corticalleptomeningeal area of enhancement was also noted in the left middle frontal gyrus at the level of the superior frontal sulcus. T2* GRE revealed no hypointense lesions consistent with hemorrhagic lesions. Brain MRI angiography showed no alterations of intracranial vessels. In the following days, the patient remained confused and demonstrated severe behavioral abnormalities, including delirium and aggressiveness. Cerebrospinal fluid analysis demonstrated normal protein content $(0.5 \mathrm{~g} / \mathrm{L}), 0$ leukocyte, and normal IgG index. A comprehensive autoimmune screening was unrevealing. On day 10, a new EEG excluded non-convulsive status epilepticus, while showing persistence of sharp slow waves, mainly over the left hemispheric regions. At the last follow-up, 14 days after admission, patient's neurological conditions were only partially improved $(\mathrm{mRS}=4)$.

\section{Case 5}

A 57-year-old man presented with fever and cough for a week with associated dyspnea for 3 days. He had a previous history of hypertension and thrombocytosis. Treatment included acetylsalicylic acid, hydroxyurea, and ramipril. Body temperature at admission was $38.4{ }^{\circ} \mathrm{C}$. Clinical and laboratory evaluation showed moderate respiratory distress $\left(\mathrm{PaO}_{2} / \mathrm{FiO}_{2}\right.$ 191). Chest $\mathrm{CT}$ demonstrated diffuse bilateral ground-glass opacities with no pleural effusion (Fig. 3a). The respiratory function progressively worsened during the following days and, on day 3 , he was transferred to ICU for invasive ventilation. The patient received $4000 \mathrm{IU}$ enoxaparin daily subcutaneously for prevention of venous thromboembolic disease. On day 4 , he developed kidney failure (creatinine $3.24 \mathrm{mg} / \mathrm{dL}$ ) treated with veno-venous hemofiltration (CVVH). Indices of inflammation and hepatic injury were increased (CRP $214 \mathrm{mg} / \mathrm{L}$, n.v. < 5; LDH 1013 U/L, n.v. 125-220; AST 97 U/L, n.v.<35; GGT 454 U/L, n.v. 8-61). 11 days after admission, the patient was found with bilaterally fixed and dilated pupils and a GCS of 3. Coagulation tests revealed prolonged aPTT (53.1 s, n.v. 23.5-35.0)
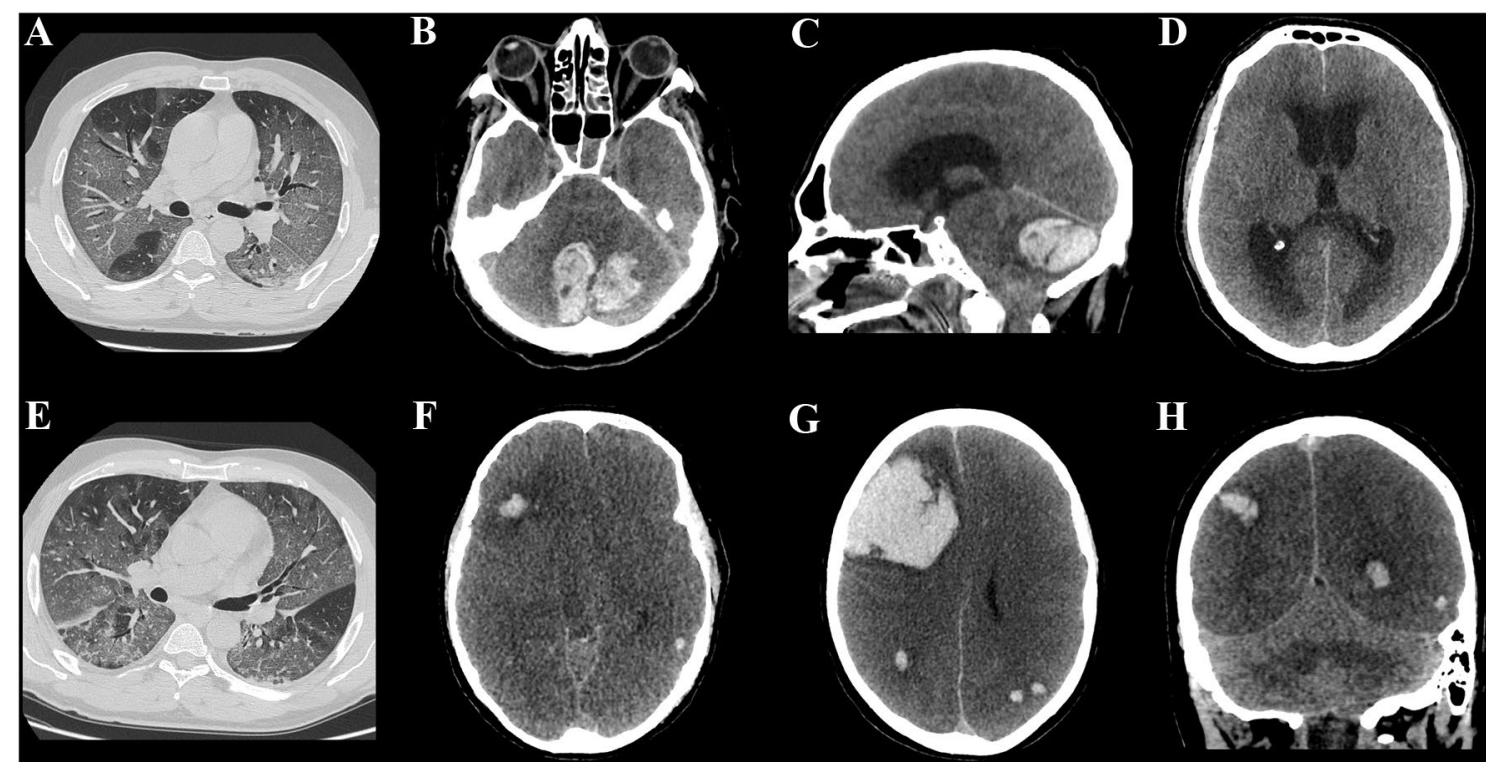

F
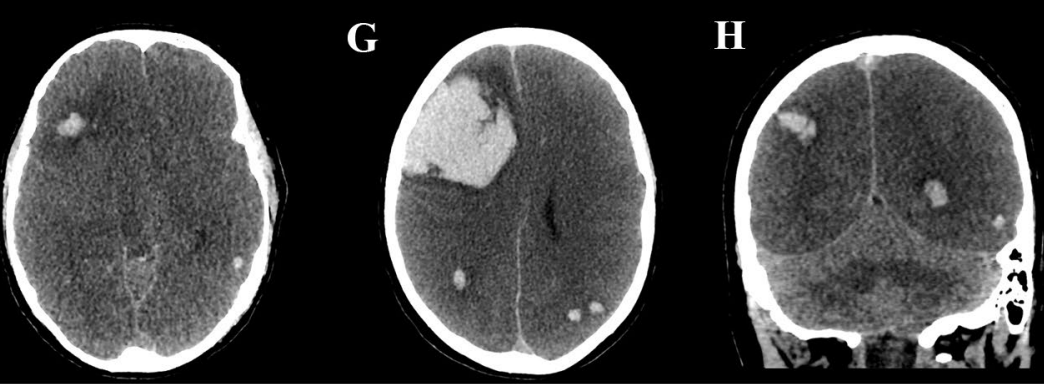

Fig. 3 Chest and brain CT examination of Patient 5 (a-d), showing diffuse bilateral ground-glass opacities involving both lungs (a), a large cerebellar hemorrhage (b) which compresses the brainstem and the fourth ventricle determining a subsequent obstructive hydrocephalus $(\mathbf{c}, \mathbf{d})$. Chest and brain CT examination of Patient $6(\mathbf{e}-\mathbf{h})$, demonstrating diffuse bilateral ground-glass opacities with no pleural effusion (e), diffuse cerebral edema with loss of normal gray-white matter differentiation and obliteration of CSF spaces (f), a large right frontal hemorrhage in association with other smaller hemorrhages on the axial and on the coronal multiplanar reconstruction of brain CT $(\mathbf{g}, \mathbf{h})$, and a bright spot within the sagittal sinus suspected for dural sinus thrombosis 
and increased D-dimer (2866 ng/mL, n.v. 0-500). A brain CT demonstrated hemorrhages (Fig. 3b, c) in both cerebellar hemispheres (diameter of $4.8 \mathrm{~cm}$ on right, and $3.6 \mathrm{~cm}$ on the left) with compression of the fourth ventricle and the brainstem. Supratentorial hydrocephalus and diffuse obliteration of sulci were also noted (Fig. 3d). CTA did not support the possibility of venous infarction due to non-opacification of the venous vessels. One hour after CT examination, the patient died.

\section{Case 6}

A previously healthy 57-year-old man presented with cough and fever for 10 days with associated dyspnea for 4 days. Body temperature at admission was $37.5^{\circ} \mathrm{C}$. Clinical and laboratory evaluation showed respiratory insufficiency $\left(\mathrm{PaO}_{2} / \mathrm{FiO}_{2}\right.$ 197), increased CRP $(21 \mathrm{mg} / \mathrm{L}$, n.v. $<5)$, and alteration of the hepatic function (LDH $771 \mathrm{U} / \mathrm{L}$, n.v. 125-220; AST 100 U/L, n.v. 4-40; GGT 152 U/L, n.v. 8-61). Chest CT demonstrated diffuse bilateral groundglass opacities with no pleural effusion (Fig. 3e). On day 5, his respiratory function worsened and he was transferred to ICU for invasive ventilation. He was treated with $4000 \mathrm{IU}$ enoxaparin daily subcutaneously. Despite regular cardiac activity and well-supported respiratory function, 7 days after admission in ICU his neurological conditions suddenly deteriorated. The patient was found with bilaterally fixed and dilated pupils, with a GCS of 3. A brain CT (Fig. 3f-h) demonstrated diffuse cerebral edema with a large right frontal hemorrhage $(6.0 \times 5.2 \mathrm{~cm}$ on the axial plane) extending to the ventricular system. The ventricles were displaced across the midline. Four additional smaller intra-axial hemorrhages occurred in both hemispheres. Coagulation tests were normal. Shortly after CT examination, the patient died.

\section{Discussion}

We report the clinical and neuroimaging features of six patients with severe SARS-CoV-2 infection who developed acute stroke, four ischemic and two hemorrhagic.

At the time the cerebrovascular complications ensued, all but one patient needed critical care support and most of them had evidence of multiorgan failure. Most of the patients were elderly male with comorbidities. Ischemic lesions were typically multiple and bilateral, suggesting an embolic etiology. Conversely, the detection of simultaneous multiple intracerebral hemorrhages associated with severe brain edema is unprecedented in this setting and raises important issues in the management of patients with Covid-19. Taken together, these factors could account for the poor outcome observed in this cohort. Given the fact that vascular risk factors were frequent, the association between SARS-CoV-2 and stroke might be coincidental. Nevertheless, a body of epidemiological, clinical and experimental evidence suggests that patients with COVID-19 are at higher risk of thromboembolic complications, including neurovascular diseases, in the acute phase of infection. In particular, previous experiences from other coronavirus-related diseases, the 2003 SARS epidemic and the 2012 Middle East respiratory syndrome (MERS), have highlighted stroke, pulmonary embolism and myocardial infarction as possible complications, especially in critically ill patients [5, 8]. Additionally, a recent report from Wuhan, China, showed that acute cerebrovascular disease is not uncommon in COVID-19, and it represents a negative prognostic factor. Among 221 infected patients, 11 (5\%) developed ischemic stroke, $1(0.5 \%)$ cerebral venous thrombosis, and $1(0.5 \%)$ brain hemorrhage [9]. In agreement with the finding of the present study, it was also observed that cardiovascular risk factors were common, while the patients demonstrated increased biomarkers of inflammatory response as well as a hypercoagulable state [9].

The relationship between acute infection and stroke is complex and it was demonstrated for many types of bacterial and viral infections. Importantly, an increased risk of stroke was documented after respiratory infections, including influenza, and the risk was highest within the first week [10]. Accordingly, measures to prevent viral illnesses (e.g., influenza vaccination) were shown to be protective for the development of cerebrovascular disease [11]. Intriguingly, the association between infection and stroke is also relevant for patients with preexistent risk factors. In this group, the vulnerability of atherosclerotic plaques to rupture appears to be increased in the presence of systemic inflammation and sepsis [10]. The mechanisms by which viral infections can trigger stroke are multiple and depend on the associated pathogen and host characteristics. For example, varicella zoster virus (VZV) is responsible for a distinctive vasculopathy involving both large and small arteries, which can result in ischemic infarction of the brain and spinal cord, as well as subarachnoid and cerebral hemorrhage [12]. At autopsy, the cerebral arteries showed disruption of the internal elastic lamina and multinucleated giant cells, while the presence of VZV DNA was demonstrated in the posterior cerebral and basilar arteries [13]. Similarly, human immunodeficiency virus (HIV) is a known cause of large vessel vasculopathy (mainly aneurysmal) that can also involve the brain [14]. A vasculitic-like mechanism can also apply in the context of COVID-19, since localized fibrinoid necrosis as well as infiltration of monocytes, lymphocytes, and plasma cells into vessel walls were demonstrated in three cases of the 2003 SARS disease [15]. Interestingly enough, another type of coronavirus (New Haven $\mathrm{CoV}$ ) was associated with the development of systemic vasculitis of childhood (Kawasaki disease) in a case-control study [16]. An alternative mechanism leading to stroke during the course of an infection is the presence of a 
pro-thrombotic state. Numerous reports have documented various coagulation abnormalities (in particular, raised D-dimer, thrombocytopenia, and isolated prolonged aPTT) in patients with SARS-CoV, nearly two decades ago [17, 18]. Chong et al. studied an autopsy series of eight SARS patients and demonstrated the presence of pulmonary embolism in half of them, while one patient developed non-bacterial valvular vegetations associated with infarctions of the heart, kidneys, spleen, and occipital lobe [18]. Similarly, an underlying thrombophilic state can also be hypothesized in SARS-CoV-2 infection, as suggested by a report of pulmonary embolism in patients from Wuhan [19] and in Patient 1 of the present series who also presented brain and spleen lesions suggestive for embolization.

We observed a marked elevation of a ubiquitous cell enzyme (LDH) in all patients of the present series. Elevated serum LDH is associated with numerous clinical conditions, including hemolysis, cancer, and sepsis [20]. In the setting of COVID-19, it is possible that the very high LDH levels reflect the underlying respiratory failure and systemic shock. Its role as a potential prognostic factor in COVID-19-associated stroke should be further investigated.

No patient with ischemic stroke was treated with thrombolysis or thrombectomy in the present series. This could be related to the fact that the patients were critically ill, and the detection of changes in neurological status is highly complex in ICU patients, where lightening of sedation and frequent neurological follow-up are needed. All these measures can be less prompt in the presence of restricted resources and limited medical personnel, as in a catastrophic medical emergency like COVID-19.

Finally, one patient (Case 4) developed an encephalopathy, characterized by focal seizures in association with behavioral abnormalities, which preceded stroke, and complicated hospital stay. No clear structural, autoimmune, or metabolic etiology was found. Given the fact that cases of encephalitis and encephalopathy linked to COVID-19 were recently reported [21], we cannot exclude that it was due to direct neuro-invasion of SARS-CoV-2, although we are unable to prove this hypothesis.

In conclusion, acute stroke can complicate the course of COVID-19 infection.

Both ischemic and hemorrhagic lesions are possible, further underscoring the delicate equilibrium between thrombophilic state and coagulopathy in patients with SARS-CoV-2. In our series, stroke developed mostly in patients with severe pneumonia and multiorgan failure, liver enzymes and lactate dehydrogenase were markedly increased in all cases, and the outcome was poor.

\section{Data access, responsibility, and analysis}

The corresponding author had full access to all the data in the study and takes responsibility for the integrity of the data and the accuracy of the data analysis.

Author contributions Study concept and design: MM, DB, AV. Acquisition of data: MM, DB. Analysis and interpretation of data: MM, DB, MC, SDA, GG, CB, AV. Drafting of the manuscript: AV, MM, DB. Critical revision of the manuscript for important intellectual content: MM, DB, MC, SDA, GG, CB, AV. Study supervision: MM. All authors read and approved the final manuscript.

Funding No targeted funding reported.

\section{Compliance with ethical standards}

Conflicts of interest The authors declare that they have no conflicts of interest.

Ethical standards All procedures were performed in accordance with the institutional ethics committee and the Declaration of Helsinki.

\section{References}

1. COVID-19 Map (2020) In: Johns Hopkins coronavirus resource center. https://coronavirus.jhu.edu/map.html. Accessed 6 Apr 2020

2. Chen N, Zhou M, Dong X et al (2020) Epidemiological and clinical characteristics of 99 cases of 2019 novel coronavirus pneumonia in Wuhan, China: a descriptive study. Lancet 395:507-513. https://doi.org/10.1016/S0140-6736(20)30211-7

3. Guan W-J, Ni Z-Y, Hu Y et al (2020) Clinical Characteristics of Coronavirus Disease 2019 in China. N Engl J Med. https://doi. org/10.1056/NEJMoa2002032

4. Wang D, Hu B, Hu C et al (2020) Clinical characteristics of 138 hospitalized patients with 2019 novel coronavirus-infected pneumonia in Wuhan, China. JAMA. https://doi.org/10.1001/ jama.2020.1585

5. Umapathi T, Kor AC, Venketasubramanian N et al (2004) Large artery ischaemic stroke in severe acute respiratory syndrome (SARS). J Neurol 251:1227-1231. https://doi.org/10.1007/s0041 5-004-0519-8

6. Khosravani H, Rajendram P, Notario L et al (2020) Protected code stroke: hyperacute stroke management during the coronavirus disease 2019 (COVID-19) pandemic. Stroke. https://doi.org/10.1161/ STROKEAHA.120.029838

7. Mortelliti MP, Manning HL (2002) Acute respiratory distress syndrome. Am Fam Physician 65:1823-1830

8. Arabi YM, Harthi A, Hussein J et al (2015) Severe neurologic syndrome associated with Middle East respiratory syndrome corona virus (MERS-CoV). Infection 43:495-501. https://doi. org/10.1007/s15010-015-0720-y

9. Li Y, Wang M, Zhou Y et al (2020) Acute cerebrovascular disease following COVID-19: a single center, retrospective, observational study. Social Science Research Network, Rochester

10. Emsley HCA, Hopkins SJ (2008) Acute ischaemic stroke and infection: recent and emerging concepts. Lancet Neurol 7:341353. https://doi.org/10.1016/S1474-4422(08)70061-9 
11. Lavallée P, Perchaud V, Gautier-Bertrand M et al (2002) Association between influenza vaccination and reduced risk of brain infarction. Stroke 33:513-518. https://doi.org/10.1161/hs020 2.102328

12. Gilden D, Cohrs RJ, Mahalingam R, Nagel MA (2009) Varicella zoster virus vasculopathies: diverse clinical manifestations, laboratory features, pathogenesis, and treatment. Lancet Neurol 8:731-740. https://doi.org/10.1016/S1474-4422(09)70134-6

13. Gilden DH, Kleinschmidt-DeMasters BK, Wellish M et al (1996) Varicella zoster virus, a cause of waxing and waning vasculitis: the New England Journal of Medicine case 5-1995 revisited. Neurology 47:1441-1446. https://doi.org/10.1212/wnl.47.6.1441

14. Chetty R, Batitang S, Nair R (2000) Large artery vasculopathy in HIV-positive patients: another vasculitic enigma. Hum Pathol 31:374-379. https://doi.org/10.1016/s0046-8177(00)80253-1

15. Ding Y, Wang H, Shen $\mathrm{H}$ et al (2003) The clinical pathology of severe acute respiratory syndrome (SARS): a report from China. J Pathol 200:282-289. https://doi.org/10.1002/path.1440

16. Esper F, Shapiro ED, Weibel C et al (2005) Association between a novel human coronavirus and Kawasaki disease. J Infect Dis 191:499-502. https://doi.org/10.1086/428291
17. Wong RSM, Wu A, To KF et al (2003) Haematological manifestations in patients with severe acute respiratory syndrome: retrospective analysis. BMJ 326:1358-1362. https://doi.org/10.1136/ bmj.326.7403.1358

18. Chong PY, Chui P, Ling AE et al (2004) Analysis of deaths during the severe acute respiratory syndrome (SARS) epidemic in Singapore: challenges in determining a SARS diagnosis. Arch Pathol Lab Med 128:195-204. https://doi.org/10.1043/15432165(2004)128\%3c195:AODDTS\%3e2.0.CO;2

19. COVID-19 (2020) Complicated by acute pulmonary embolism/radiology: cardiothoracic imaging. https://pubs.rsna. org/doi/10.1148/ryct.2020200067. Accessed 8 Apr 2020

20. Erez A, Shental O, Tchebiner JZ et al (2014) Diagnostic and prognostic value of very high serum lactate dehydrogenase in admitted medical patients. Isr Med Assoc J 16:439-443

21. Moriguchi T, Harii N, Goto J et al (2020) A first case of meningitis/encephalitis associated with SARS-Coronavirus-2. Int J Infect Dis. https://doi.org/10.1016/j.ijid.2020.03.062 